\title{
L-Theanine promotes cultured human Sertoli cells proliferation and modulates glucose metabolism
}

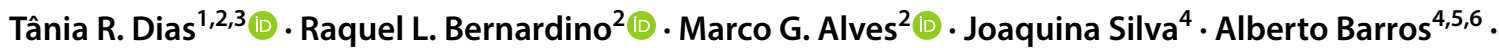 \\ Mário Sousa $^{2,4} \cdot$ Susana Casal ${ }^{3}$ (1) $\cdot$ Branca M. Silva ${ }^{1}$ (D) Pedro F. Oliveira ${ }^{2,5,6}$ (])
}

Received: 11 September 2018 / Accepted: 14 May 2019 / Published online: 10 June 2019

○) Springer-Verlag GmbH Germany, part of Springer Nature 2019

\begin{abstract}
Purpose L-Theanine is the major free amino acid present in tea (Camellia sinensis L.). The effects of several tea constituents on male reproduction have been investigated, but L-theanine has been overlooked. Sertoli cells (SCs) are essential for the physical and nutritional support of germ cells. In this study, we aimed to investigate the ability of L-theanine to modulate important mechanisms of human SCs (hSCs) metabolism, mitochondrial function and oxidative profile, which are essential to prevent or counteract spermatogenesis disruption in several health conditions.

Methods We evaluated the effect of a dose of L-theanine attained by tea intake $(5 \mu \mathrm{M})$ or a pharmacological dose $(50 \mu \mathrm{M})$ on the metabolism (proton nuclear magnetic resonance and Western blot), mitochondrial functionality (protein expression of mitochondrial complexes and JC1 ratio) and oxidative profile (carbonyl levels, nitration and lipid peroxidation) of cultured hSCs.

Results Exposure of hSCs to $50 \mu \mathrm{M}$ of L-theanine increased cell proliferation and glucose consumption. In response to this metabolic adaptation, there was an increase in mitochondrial membrane potential, which may compromise the prooxidantantioxidant balance. Still, no alterations were observed regarding the oxidative damages.

Conclusions A pharmacological dose of L-theanine $(50 \mu \mathrm{M})$ prompts an increase in hSCs proliferation and a higher glucose metabolization to sustain the pool of Krebs cycle intermediates, which are crucial for cellular bioenergetics and biosynthesis. This study suggests an interplay between glycolysis and glutaminolysis in the regulation of hSCs metabolism.
\end{abstract}

Keywords Cell metabolism $\cdot$ Glutamate $\cdot \mathrm{L}-$ Theanine $\cdot$ Mitochondria $\cdot$ Antioxidant $\cdot$ Sertoli cell

Branca M. Silva

bmcms@gmail.com

Pedro F. Oliveira

pfobox@gmail.com

Universidade da Beira Interior, 6201-001 Covilhã, Portugal

2 Department of Microscopy, Laboratory of Cell Biology and Unit for Multidisciplinary Research in Biomedicine, Institute of Biomedical Sciences Abel Salazar (ICBAS), University of Porto, 4050-313 Porto, Portugal

3 LAQV/REQUIMTE, Laboratory of Bromatology and Hydrology, Faculty of Pharmacy, University of Porto, 4050-313 Porto, Portugal

4 Centre for Reproductive Genetics Prof. Alberto Barros, 4100-009 Porto, Portugal

5 Department of Genetics, Faculty of Medicine, University of Porto, 4050-313 Porto, Portugal

6 i3S, Instituto de Investigação e Inovação em Saúde, Universidade do Porto, 4200-135 Porto, Portugal

\begin{tabular}{ll}
\multicolumn{2}{l}{ Abbreviations } \\
4-HNE & 4-Hydroxynonenal \\
AP & Alkaline phosphatase \\
BBB & Blood-brain barrier \\
BTB & Blood-testis barrier \\
DNP & 2,4-Dinitrophenyl \\
EGCG & Epigallocatechin gallate \\
FBS & Fetal bovine serum \\
hSCs & Human Sertoli cells \\
ITS & Insulin-transferrin-sodium selenite \\
LDH & Lactate dehydrogenase \\
MCT4 & Monocarboxylate transporter 4 \\
NMR & Nuclear magnetic resonance \\
PBS & Phosphate-buffered saline \\
PVDF & Polyvinylidene difluoride \\
ROS & Reactive oxygen species \\
SCs & Sertoli cells \\
SEM & Standard error of the mean \\
SRB & Sulforhodamine B
\end{tabular}




\section{Introduction}

Tea (Camellia sinensis L.) consumption has been associated with a wide variety of health benefits, thus encouraging many people to include it in their daily alimentary routine. Although tea composition is highly variable according to the type (white, green, oolong or black) and geographical origin, the most bioactive tea compounds include methylxanthines, phenolic compounds and amino acids. L-theanine ( $\mathrm{L}-\boldsymbol{\gamma}$-glutamylethylamide) is a nonproteinogenic amino acid almost exclusively found in this botanical species. L-theanine is usually associated with tea unique taste and relaxation properties. For that reason, it has gained popularity and become a common ingredient in functional beverages and food supplements.

Human reproduction is highly reliant on the success of sperm production in males. This complex process known as spermatogenesis is mainly sustained by Sertoli cells (SCs), which are the somatic cells present in the seminiferous tubules of the testes. These cells constitute the blood-testis-barrier (BTB), which is crucial for the physical support and protection of developing germ cells. They are also responsible for the production of several growth factors and nutrients, essential for germ cell survival and differentiation into spermatozoa [1]. The normal function of SCs metabolic processes is crucial for the preservation of male reproductive potential. These cells produce high amounts of lactate, which is then used as an energy source by the developing germ cells. The main substrate used by $\mathrm{SCs}$ to produce lactate is glucose, but they can also metabolize amino acids such as glutamine.

Several pathological conditions, such as diabetes mellitus and obesity, may trigger severe alterations in SCs metabolism, thus disturbing spermatogenesis and compromising male fertility. In fact, the increasing incidence of those diseases around the world has been accompanied by a decrease in male reproductive health. This scenario highlighted the need to find new modulators of SCs metabolism to prevent the negative effects of those diseases on male reproduction. Studies from our team demonstrated that tea and some of its constituents [e.g., caffeine and epigallocatechin gallate (EGCG)] can modulate SC function in vitro [2-4] and improve male reproductive potential in vivo [5]. However, to our knowledge, there are no studies reporting the effects of L-theanine on SC function. L-theanine has demonstrated a preventive role against oxidative stress in other cell types, such as hepatocytes [6] or neurons $[7,8]$. One of the major concerns regarding SCs functions is that alterations in their metabolism can compromise the prooxidant-antioxidant homeostasis due to excessive production of reactive oxygen species (ROS). In this study, we aimed to investigate the ability of L-theanine to modulate important mechanisms of hSCs metabolism, mitochondrial function and oxidative profile, which are essential to prevent or counteract spermatogenesis disruption in several health conditions.

\section{Materials and methods}

\section{Chemicals}

The following chemicals were used-fetal bovine serum (FBS; S0615): Biochrom (Leonorenstr, Berlin, Germany); insulin-transferrin-selenium (ITS; 41400045): Gibco ${ }^{\text {TM }}$ by Life Technologies (Carlsbad, California, USA); L-theanine (ab141187; CAS number 3081-61-6) and extracellular $\mathrm{O}_{2}$ consumption assay (ab197243): Abcam (Cambridge, UK); Tris base (MB01601) NZYTech (Lisbon, Portugal); M-PER Mammalian Protein Extraction Reagent (78501) and LDH Enzymatic Assay Kit (88954): Thermo Scientific (Waltham, MA, USA); JC1 (T3168): Molecular Probes (Eugene, OR, USA); All other chemicals: Sigma-Aldrich (St. Louis, MO, USA) unless specifically stated.

\section{Cell primary cultures and experimental groups}

The processing of human testicular tissue was performed at Centre for Reproductive Genetics Professor Alberto Barros (Porto, Portugal) according to local, national, and European ethical committees' guidelines and the Declaration of Helsinki. Six testicular biopsies were obtained from six different patients with conserved spermatogenesis, after informed written consent. hSCs were obtained from the cells left in tissue culture plates after each patient's treatment. Cells from each individual were plated separately in six cell + culture flasks (Sarstedt, Nümbrecht, Germany) and incubated at $33-34{ }^{\circ} \mathrm{C}$, in a controlled atmosphere of $5 \% \mathrm{CO}_{2}$. The purity of hSCs culture was assessed by the immunoperoxidase technique. Briefly, cells were incubated with primary polyclonal antibody and processed by the labeled streptavidin-biotin method using an ExtrAvidin Peroxidase Staining Kit (Sigma-Aldrich). Besides, specific protein markers, the anti-Mullerian hormone and vimentin, were also used to assess the purity of hSCs cultures [9]. After $96 \mathrm{~h}$, cultures were examined by phase contrast microscopy and only hSCs with other cell contaminants below $5 \%$ were used.

Cells were left to grow until reach $90-95 \%$ confluence and cells from each flask were divided into three culture plates (Bioportugal, Porto, Portugal), making a total of 18 plates. After reaching 90-95\% confluence, the culture media of the culture plates were replaced by serum-free media (DMEM:F12) supplemented with ITS medium $(10 \mu \mathrm{g} / \mathrm{mL}$ insulin-5.5 $\mu \mathrm{g} / \mathrm{mL}$ transferrin-0.0067 $\mu \mathrm{g} / \mathrm{mL}$ sodium selenite). Three experimental groups were defined: a control 
group (without L-theanine) and two groups supplemented either with 5 or $50 \mu \mathrm{M}$ of L-theanine. Previous studies reported that the ingestion of $25-100 \mathrm{mg}$ of L-theanine (either as tea or capsules) corresponded to a concentration of 5-25 $\mu \mathrm{M}$ in human plasma [10]. However, the beneficial health effects of L-theanine are mostly ascribed to higher doses of $200 \mathrm{mg}$, which correspond to a bioavailability of $50 \mu \mathrm{M}$. Thus, we decided to use 5 and $50 \mu \mathrm{M}$ in this study. Besides, this will also allow the comparison with previously studied tea components, EGCG and caffeine, using this in vitro model $[2,3]$. After $24 \mathrm{~h}$ of treatment with 0 (no L-theanine), 5 or $50 \mu \mathrm{M}$ of L-theanine, cells and the respective culture media were collected. This time point was defined based on the fact that in serum, the concentration of L-theanine begins to drop slowly within $24 \mathrm{~h}$ [10]. Trypan blue exclusion test was performed to guarantee cellular integrity after the $24 \mathrm{~h}$ treatment, which averaged $85-90 \%$.

\section{Sulforhodamine B assay}

A sulforhodamine B (SRB) colorimetric assay was performed as previously described [3], to evaluate hSCs' proliferative responses to the $24 \mathrm{~h}$ exposure to 0,5 or $50 \mu \mathrm{M}$ of L-theanine. A blank was made with Tris base (pH 10) and the absorbance was read at $492 \mathrm{~nm}$. Absorbance readings of SRB-stained cells give a direct measure of cell numbers. To obtain concentration-response curves, we defined the cell growth of the control group as $100 \%$ and calculated the cell growth of treated groups relative to control.

\section{MTT reduction}

To evaluate hSCs' metabolic viability after exposure to L-theanine, we measured the reduction of MTT (3-(4,5-dimethylthiazol-2-yl)-2,5-diphenyltetrazolium bromide) tetrazolium to produce a purple formazan product. In brief, hSCs were cultured in a 96-well culture plate with DMEM:F12 supplemented with 10\% FBS. After reaching 90-95\% confluence, hSCs were exposed to ITS medium supplemented with 0,5 or $50 \mu \mathrm{M}$ of L-theanine for $24 \mathrm{~h}$. Then, the medium was removed and replaced by $150 \mu \mathrm{L}$ of freshly prepared ITS medium. MTT was firstly dissolved at $5 \mathrm{mg} /$ $\mathrm{mL}$ in warm PBS $1 \times$ and protected from light. $15 \mu \mathrm{L}$ of MTT solution was added to each well to attain a final concentration of $0.5 \mathrm{mg} / \mathrm{mL}$ per well and cells were incubated during $3 \mathrm{~h}$ at $37^{\circ} \mathrm{C}$. At the end of incubation, the media were removed and formazan crystals were dissolved in $100 \mu \mathrm{L}$ of DMSO by gentle shaking for $10 \mathrm{~min}$ at room temperature. A blank with DMSO was made for normalization. Absorbance was recorded at $570 \mathrm{~nm}$ to quantify formazan formation (directly proportional to the number of viable cells) and also at $655 \mathrm{~nm}$ for reference. hSCs' metabolic viability was expressed in fold variation to control.

\section{LDH leakage and intracellular enzymatic activity}

To evaluate the possible cytotoxicity of L-theanine to hSCs, we assessed lactate dehydrogenase (LDH) leakage (from damaged or destroyed cells) into the extracellular fluid and intracellular LDH activity levels after $24 \mathrm{~h}$ of exposure to the experimental doses. LDH levels were spectrophotometrically determined using the LDH Enzymatic Assay Kit according to the manufacturer's instructions. For LDH leakage, $50 \mu \mathrm{L}$ of extracellular medium was mixed with $50 \mu \mathrm{L}$ of $\mathrm{LDH}$ assay substrate and incubated at room temperature for $30 \mathrm{~min}$. Then, $50 \mu \mathrm{L}$ of LDH stop solution was added to stop the enzymatic activity and absorbance was measured at $490 \mathrm{~nm}$ and $630 \mathrm{~nm}$ using an Anthos 2010 microplate reader (Biochrom, Berlin, Germany). Results were normalized to the blank and expressed as fold variation to the control group. LDH intracellular activity was evaluated as previously described [2]. LDH enzymatic activities were calculated as units per milligram of protein using the molar extinction factor $(\varepsilon)$ and final expressed as fold variation to the control group.

\section{Autophagy}

hSCs were grown in DMEM:F12 supplemented with $10 \%$ FBS, in 96-well plates until reaching $80-90 \%$ confluence. After treatment with 0,5 or $50 \mu \mathrm{M}$ of L-theanine for $24 \mathrm{~h}$, the media were removed and cells were washed with PBS. Then, cells were incubated with $100 \mu \mathrm{L}$ of propidium iodide $(1 \mu \mathrm{g} / \mathrm{mL}$ in PBS) at room temperature for $2 \mathrm{~min}$. Cells were washed again with PBS and incubated with $100 \mu \mathrm{L}$ of dansylcadaverine $\left(1 \mu \mathrm{g} / \mathrm{mL}\right.$ in PBS) at $37{ }^{\circ} \mathrm{C}$ for $10 \mathrm{~min}$. Afterward, cells were washed with PBS and incubated with $100 \mu \mathrm{L}$ of Hoechst $(10 \mu \mathrm{g} / \mathrm{mL}$ in PBS) at room temperature for $10 \mathrm{~min}$. During the incubation times, the 96-well plate was protected from light to avoid loss of fluorescence. After washing again, fresh PBS was added to each well and fluorescence was immediately analyzed using a Synergy HT Multi-Mode Microplate Reader (BioTek, Winooski, USA) pre-heated at $37{ }^{\circ} \mathrm{C}$. Death cells stained with propidium iodide were detected at $530 \pm 25 / 590 \pm 35 \mathrm{~nm}$ (excitation/ emission), autophagic cells stained with dansylcadaverine were detected at $360 \pm 40 / 528 \pm 20 \mathrm{~nm}$ (excitation/emission) and cell nucleus stained with Hoechst were detected at $360 \pm 40 / 460 \pm 40$ (excitation/emission). Results were normalized to the number of cells and expressed as fold variation to the control group. A positive control of DMSO 10\% was used for test validity.

\section{Proton nuclear magnetic resonance ('H-NMR) spectroscopy}

Culture medium was collected before hSCs treatment $(0 \mathrm{~h})$ and after the $24 \mathrm{~h}$ of treatment with $\mathrm{L}$-theanine, to allow 
the analysis of metabolites production/consumption during that incubation period. ${ }^{1} \mathrm{H}-\mathrm{NMR}$ spectra of hSCs extracellular culture media were acquired and quantified using the previously described conditions [5]. Sodium fumarate (final concentration of $1 \mathrm{mM}$ ) was used as an internal reference $(6.50 \mathrm{ppm})$ to quantify the following metabolites present in hSCs extracellular media (multiplet, ppm): lactate (doublet, 1.33); alanine (doublet, 1.45) and $\mathrm{H} 1-\alpha$-glucose (doublet, 5.22). Relative areas of ${ }^{1} \mathrm{H}-\mathrm{NMR}$ resonances and metabolite concentrations were quantified as described [5]. Results are presented as metabolite consumption or production in $\mathrm{pmol} / \mathrm{cell}$.

\section{Western blot}

Total protein fraction from hSCs was isolated using M-PER Mammalian Protein Extraction Reagent according to the manufacturer's instructions. Proteins were fractionated in $12 \%$ SDS-PAGE gels, then the separated proteins were transferred to previously activated polyvinylidene difluoride (PVDF) membranes and blocked for 90 min with a 5\% nonfat milk solution at room temperature. Afterward, the membranes were incubated overnight $\left(4{ }^{\circ} \mathrm{C}\right)$ with rabbit antimonocarboxylate transporter 4 (MCT4) (1:1000, sc-50329, Santa Cruz Biotechnology, Heidelberg, Germany), rabbit anti-lactate dehydrogenase (LDH) (1:10,000, ab52488, Abcam, Cambridge, UK) or mouse total OXPHOS cocktail (1:1000, ab110413, Abcam, Cambridge, UK) primary antibodies. Mouse anti- $\beta$-actin (1:5000, MA5-15739, Thermo Scientific, Waltham, MA, USA) was used as the protein loading control. The immunoreactive proteins were detected separately and visualized after incubation (90 $\mathrm{min}$ at room temperature) with the respective secondary antibodies: goat anti-rabbit IgG-alkaline phosphatase (AP) (1:5000, A3687) or goat anti-mouse IgG-AP (1:5000, A3562). Membranes were reacted with ECFTM (GE, Healthcare, Buckinghamshire, UK) and read with the Bio-Rad FX-Pro-plus (BioRad, Hemel Hempstead, UK). Densities from each band were obtained with BIO-PROFIL Bio-1D Software from Quantity One (Vilber Lourmat, Marne-la-Vallée, France) according to standard methods. The band density attained was divided by the corresponding $\beta$-actin band intensities and expressed as fold variation (induction/reduction) relative to the control group.

\section{Mitochondrial membrane potential}

The fluorescent probe 5-5',6-6'-tetrachloro-1,1',3,3'tetraethylbenzimidazol-carbocyanine iodide (JC1) was used to measure the mitochondrial membrane potential of hSCs after exposure to 0,5 or $50 \mu \mathrm{M}$ of L-theanine, as previously described [3]. The accumulation of the JC1 dye in mitochondria depends upon the mitochondrial membrane potential.
Fluorescence intensities were analyzed immediately using a Cytation ${ }^{\mathrm{TM}} 3$ Cell Imaging Multi-Mode Reader (BioTek, Winooski, USA) pre-heated at $37^{\circ} \mathrm{C}$. Cells with functional mitochondria exhibited $\mathrm{JC} 1$ aggregates that were detected at 550/590 nm (excitation/emission), while cells with dysfunctional mitochondria mainly exhibited JC1 monomers detected at $485 / 535 \mathrm{~nm}$ (excitation/emission). The $\mathrm{JC} 1$ ratio aggregates/monomers were calculated for each condition as a measure of mitochondrial functionality. Results are presented as fold variation to the control group. A positive control with $10 \mu \mathrm{M}$ of carbonyl cyanide-4-(trifluoromethoxy) phenylhydrazone (FCCP) was used for assay validation.

\section{Extracellular oxygen consumption assay}

Oxygen consumption is one of the most informative and direct measures of mitochondrial function. We used the extracellular oxygen consumption assay kit (ab197243; Abcam, Cambridge, UK) as previously described [3], to measure the respiration of hSCs after $24 \mathrm{~h}$ exposure to 0,5 or $50 \mu \mathrm{M}$ of L-theanine. A positive control of sodium bisulfite was used for assay validation. Fluorescence intensities were analyzed immediately using a Synergy HT Multi-Mode Microplate Reader (BioTek, Winooski, USA) pre-heated at $37^{\circ} \mathrm{C}$. Extracellular oxygen consumption of hSCs was measured at $1.5 \mathrm{~min}$ intervals for $120 \mathrm{~min}$ at $380 / 650 \mathrm{~nm}$ (excitation/emission). Cells' respiration leads to oxygen depletion from the surrounding environment, resulting in the increase in fluorescence signal. Fluorescence intensities were normalized to the blank and expressed as counts per second (CPS) versus time ( $\mathrm{min})$.

\section{Analysis of carbonyl groups, nitration and lipid peroxidation}

Carbonyl groups, nitration and lipid peroxidation are usually used as biomarkers for oxidation and can be evaluated by measuring its resulting products, 2,4-dinitrophenyl (DNP), nitro-tyrosine and 4-hydroxynonenal (4-HNE), respectively. The content of these adducts in hSCs after exposure to L-theanine was evaluated by slot blot technique. The resulting PVDF membranes were incubated overnight $\left(4{ }^{\circ} \mathrm{C}\right)$ with rabbit anti-DNP (D9656, Sigma-Aldrich, St. Louis, MO, USA), rabbit anti-nitro-tyrosine (\#9691, Cell signaling Technology, Leiden, Netherlands) or goat anti-4HNE (AB5605, Merck Millipore Temecula, USA) primary antibodies (dilution 1:5000). The immunoreactive proteins were detected separately and visualized with goat antirabbit IgG-AP (1:5000, A3687) or rabbit anti-goat IgG-AP (1:5000, A4187). Results were expressed as fold variation to the control group. 


\section{Statistical analysis}

Statistical significance was assessed by a two-tailed Mann-Whitney test for independent samples, using the GraphPad Prism 6 software (GraphPad Software, San Diego, CA, USA). All data are presented as mean \pm SEM $(N=6)$. Differences with $p<0.05$ were considered statistically significant.

\section{Results}

\section{Pharmacological dose of L-theanine induces an increase in hSCs proliferation while maintaining cellular metabolic viability}

Our results showed a significant increase in hSCs proliferation after exposure to a pharmacological dose of $50 \mu \mathrm{M}$ of L-theanine (191 $\pm 17 \%)$, not only relative to the control group $(100 \pm 8 \% ; p=0.002)$ but also to the group of hSCs exposed to $5 \mu \mathrm{M}$ of L-theanine (121 $\pm 7 \% ; p=0.009)$ (Fig. 1A). Nonetheless, hSC metabolic viability, as reflected by the MTT assay, was maintained when cultured with 5 or $50 \mu \mathrm{M}$ of L-theanine $(1.09 \pm 0.14$ and $1.02 \pm 0.18$-fold variation to control, respectively) in comparison to the non-exposed hSCs (Fig. 1B). In fact, the addition of 5 or $50 \mu \mathrm{M}$ of L-theanine to the culture medium was not cytotoxic to hSCs, as demonstrated by the LDH leakage assay (Fig. 1C). The group of hSCs exposed to $5 \mu \mathrm{M}$ of $\mathrm{L}$-theanine presented an $\mathrm{LDH}$ leakage of $1.23 \pm 0.24$-fold variation to the control, a similar value to those exposed to $50 \mu \mathrm{M}$ of L-theanine $(1.27 \pm 0.17$ fold variation to control) and with no significant alterations relative to the control group (without L-theanine). Moreover, an autophagy assay was performed to evaluate if the selected doses of L-theanine could affect hSC autophagic process, but no differences were found among the experimental groups (Fig. 1D). The autophagy levels in the groups of hSCs exposed to 5 or $50 \mu \mathrm{M}$ of L-theanine were $1.07 \pm 0.06$ and $1.02 \pm 0.06$-fold variation to control, respectively.

\section{Glucose consumption by hSCs was stimulated by exposure to the pharmacological dose of L-theanine}

Our results showed that non-exposed hSCs presented a glucose consumption of $7.23 \pm 3.85 \mathrm{pmol} / \mathrm{cell}$. The addition of $5 \mu \mathrm{M}$ of L-theanine to the cell culture medium did not affect glucose consumption $(8.74 \pm 2.11 \mathrm{pmol} / \mathrm{cell})$ in comparison to the control group. On the other hand, there was an increase (to $28.55 \pm 7.24 \mathrm{pmol} / \mathrm{cell}$ ) in glucose consumption of hSCs exposed to the pharmacological dose of L-theanine $(50 \mu \mathrm{M})$, relative not only to the control group $(p=0.032)$ but also to the group of hSCs exposed to $5 \mu \mathrm{M}$ of $\mathrm{L}$-theanine $(p=0.016)$ (Fig. 2A).

\section{Lactate production was maintained after exposure to L-theanine}

Our results demonstrated that $\mathrm{LDH}$ protein levels were similar in hSCs from the control group and those cultured with 5 or $50 \mu \mathrm{M}$ of L-theanine $(1.07 \pm 0.15$ and $0.92 \pm 0.11$-fold variation to control, respectively) (Fig. 2B). Besides, LDH activity of hSCs exposed to 5 or $50 \mu \mathrm{M}$ of L-theanine was $0.74 \pm 0.12$ and $1.14 \pm 0.30$-fold variation to the control,
Fig. 1 Effect of L-theanine (5 and $50 \mu \mathrm{M}$ ) in human Sertoli cells (hSCs) survival. The figure shows pooled data of independent experiments, indicating $\mathrm{hSC}$ proliferation $(\mathbf{A})$, metabolic viability $(\mathbf{B}), \mathrm{LDH}$ cytotoxicity $(\mathbf{C})$ and autophagy (D). Cell proliferation data are presented as percentage, where the control value was set at $100 \%$, while that of metabolic viability, LDH cytotoxicity and autophagy are presented as fold variation to the control. All results are expressed as mean $\pm \operatorname{SEM}(n=6$ for each condition). Significantly different results $(p<0.05)$ are indicated as: a-relative to control; $\mathrm{b}$-relative to $5 \mu \mathrm{M}$ L-theanine
A

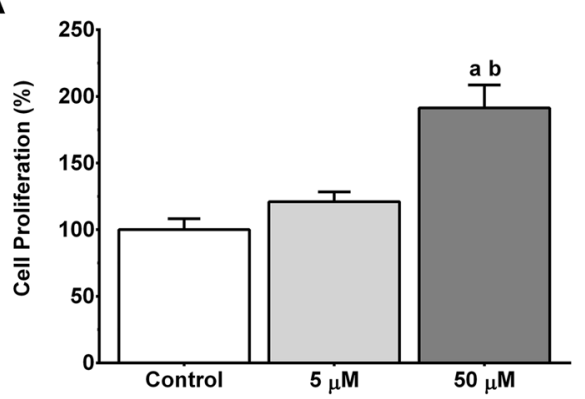

C

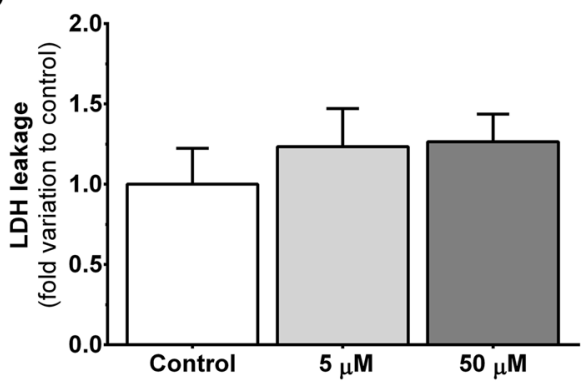

B

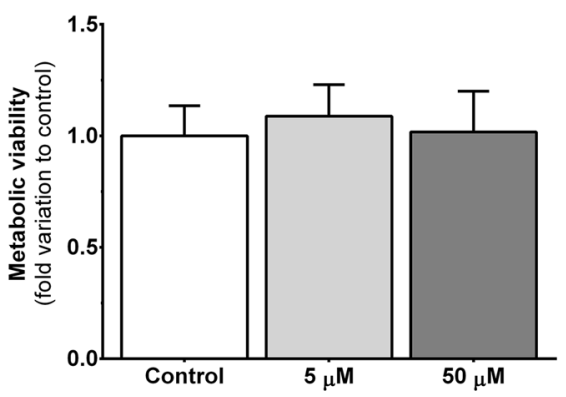

D

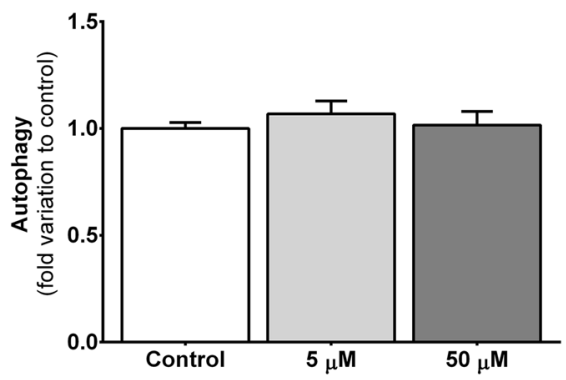


A

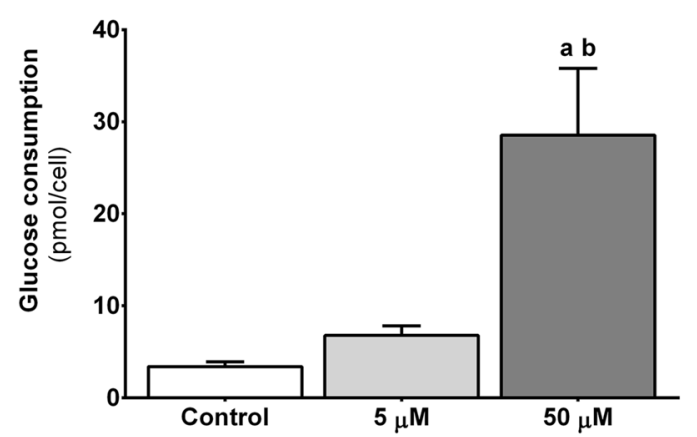

C

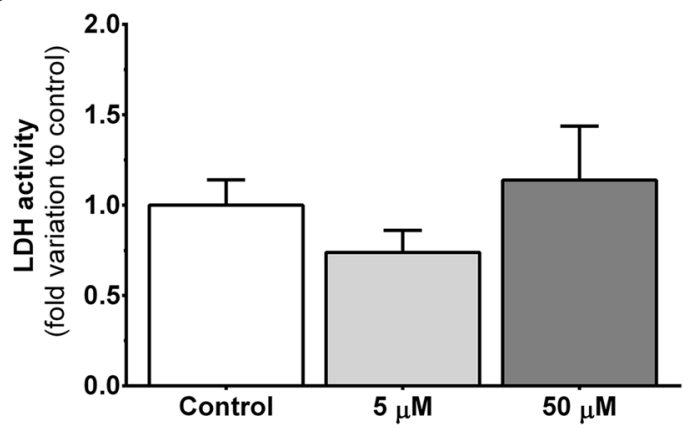

B

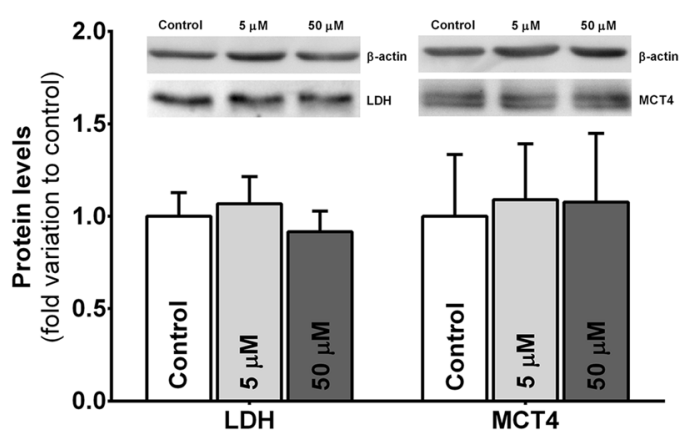

D

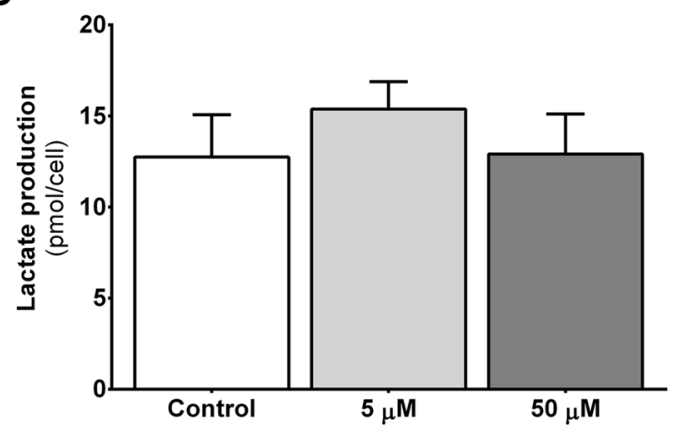

Fig. 2 Effect of L-theanine ( 5 and $50 \mu \mathrm{M})$ in glucose metabolism of human Sertoli cells (hSCs). The figure shows pooled data of independent experiments, indicating glucose consumption (A), LDH and MCT4 protein levels (B), LDH activity (C) and lactate production (D). B Also displays the representative blots (of one sample) of LDH and MCT4. While glucose consumption and lactate production are

respectively, with no significant differences to LDH activity in hSCs from the control group (Fig. 2C). Likewise, lactate production by hSCs was not altered by exposure to the selected doses of L-theanine (Fig. 2D). In fact, nonexposed hSCs produced $12.75 \pm 2.33 \mathrm{pmol} / \mathrm{cell}$ of lactate and hSCs exposed to 5 or $50 \mu \mathrm{M}$ of L-theanine produced a similar amount $(15.38 \pm 1.51$ and $12.91 \pm 2.20 \mathrm{pmol} / \mathrm{cell}$, respectively). Moreover, the protein levels of MCT4 in hSCs exposed to 5 or $50 \mu \mathrm{M}$ of $\mathrm{L}$-theanine were similar to those of the control group $(1.09 \pm 0.30$ and $1.08 \pm 0.37$-fold variation to control, respectively). In addition, alanine production by hSCs was not altered by exposure to L-theanine (data not shown).

\section{Mitochondrial membrane potential increased in $\mathrm{hSC}$ exposed to the pharmacological dose of L-theanine}

The addition of 5 or $50 \mu \mathrm{M}$ of L-theanine to hSCs culture medium did not induce significant alterations in the protein levels of mitochondrial complexes I-V (Table 1). Additionally, the established mitochondrial membrane presented as pmol/cell, results from protein levels and LDH activity are presented as fold variation to the control. All results are expressed as mean \pm SEM ( $n=6$ for each condition). Significantly different results $(p<0.05)$ are indicated as: a-relative to control; $\mathrm{b}$-relative to $5 \mu \mathrm{M}$ L-theanine

potential of hSCs was evaluated by the JC1 ratio and our results showed an increase in hSCs cultured with $50 \mu \mathrm{M}$ of L-theanine $(2.02 \pm 0.25$-fold variation to the control) relative to the control group ( $p=0.010$ ). No significant differences were found among hSCs from the control group and those exposed to $5 \mu \mathrm{M}$ of $\mathrm{L}$-theanine $(1.60 \pm 0.24$-fold variation to the control) (Fig. 3A). Nevertheless, oxygen consumption was similar among the hSCs from the several experimental groups (Fig. 3B).

\section{Exposure to L-theanine did not induce oxidative damage in hSCs}

We have measured the levels of the end products resultant from protein oxidation or nitration, as well as lipid peroxidation on hSCs after exposure to 5 or $50 \mu \mathrm{M}$ of L-theanine. Our data showed that the addition of 5 or $50 \mu \mathrm{M}$ of L-theanine to the culture medium of hSCs did not alter the levels of carbonyl groups, nitration or lipid peroxidation comparatively to the control group (Table 2). 
Table 1 Protein expression levels of mitochondrial complexes in human Sertoli cells from the control group and groups exposed to 5 or $50 \mu \mathrm{M}$ of L-theanine

\begin{tabular}{llll}
\hline $\begin{array}{l}\text { Mito- } \\
\text { chondrial } \\
\text { complexes }\end{array}$ & Control & $5 \mu \mathrm{M}$ of L-theanine & $50 \mu \mathrm{M}$ of L-theanine \\
\hline $\mathrm{CI}$ & $1.00 \pm 0.18$ & $1.07 \pm 0.06$ & $0.98 \pm 0.15$ \\
$\mathrm{CII}$ & $1.00 \pm 0.14$ & $1.13 \pm 0.17$ & $0.90 \pm 0.12$ \\
$\mathrm{CIII}$ & $1.00 \pm 0.09$ & $0.99 \pm 0.14$ & $0.85 \pm 0.06$ \\
$\mathrm{CIV}$ & $1.00 \pm 0.11$ & $1.02 \pm 0.11$ & $0.86 \pm 0.09$ \\
$\mathrm{CV}$ & $1.00 \pm 0.08$ & $1.20 \pm 0.22$ & $0.89 \pm 0.08$ \\
\hline
\end{tabular}

Results are expressed as mean \pm SEM (fold variation to the control), $n=6$ for each condition

CI NADH dehydrogenase (ubiquinone) 1 beta subcomplex subunit 8 (NDUFB8), CII Succinate dehydrogenase complex, subunit B, iron sulfur (SDHB), CIII ubiquinol-cytochrome c reductase core protein II (UQCRC2), CIV mitochondrially encoded cytochrome c oxidase I (MTCO1), $C V$ ATP synthase alpha-subunit (ATP5A)

\section{Discussion}

It was previously shown that L-theanine can have an important role in the preservation of rat sperm viability (ex vivo) when combined with EGCG and caffeine [4], but the underlying molecular mechanisms are still unknown. As SCs' function is essential for the support of spermatogenesis and male fertility, in the present study, we used an in vitro model of hSCs to unveil the effects of L-theanine in these somatic testicular cells.

L-Theanine is considered safe for humans as no toxic effects have been reported so far, though the regulation for its ingestion varies among countries. While in Japan L-theanine use in dietary products has no dose restrictions, in the USA, the Food and Drug Administration (FDA) considers it to be generally recognized as safe (GRAS), but recommends a maximum daily consumption of $1200 \mathrm{mg}$. Nevertheless, as its effects on cultured hSCs are unknown, we evaluated the effect of two doses of theanine $(5 \mu \mathrm{M}$-representing a quantity easily obtained through diet; and $50 \mu \mathrm{M}$-representing a pharmacological dose) in hSCs. Firstly, we evaluated hSC proliferation, metabolic viability, autophagy and LDH release (Fig. 1) after $24 \mathrm{~h}$ of exposure. The incubation with the lowest dose of L-theanine $(5 \mu \mathrm{M})$ did not prompt any alterations in the considered parameters, while exposure of hSCs to the pharmacological dose of L-theanine $(50 \mu \mathrm{M})$ induced an increase in cell proliferation when compared to the control group. These results suggest that the selected doses are not toxic for hSCs. Besides, as each SC can only support a limited number of germ cells [11], a higher proliferation may be important to increase spermatozoa production and improve male reproductive potential. However, we must consider that in vivo the proliferation of adult SCs is limited, so data extrapolation should be made carefully. Still,
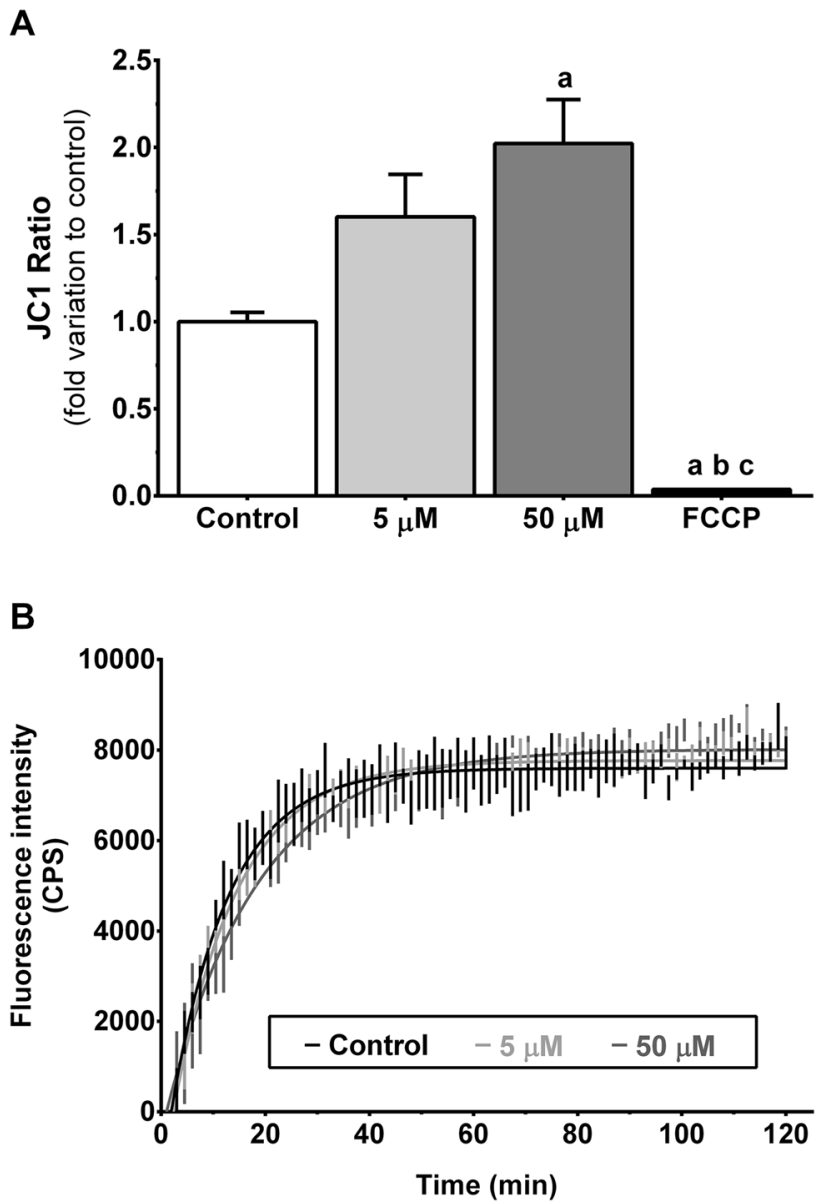

Fig. 3 Effect of L-theanine ( 5 and $50 \mu \mathrm{M})$ in mitochondrial function of human Sertoli cells (hSCs). The figure shows pooled data of independent experiments, indicating mitochondrial membrane potential (JC1 ratio) (A) and extracellular $\mathrm{O}_{2}$ consumption (B). Results are presented as fold variation to control. Results are expressed as mean \pm SEM ( $n=5$ for each condition). Significantly different results $(p<0.05)$ are indicated as: a-relative to control; b-relative to $5 \mu \mathrm{M}$ L-theanine; c-relative to $50 \mu \mathrm{M}$ L-theanine. FCCP carbonyl cyanide4-(trifluoromethoxy)phenylhydrazone

this in vitro model of proliferating hSCs is a well-established and published model for toxicological studies [2, 12-14], retaining many specific characteristics of its derived tissue [15]. A recent study also reported the pro-proliferative action of L-theanine exposure (1-100 $\mu \mathrm{M}$ for 12 days) in cultured murine neuronal progenitor cells [16], thus corroborating our observations.

Generally, high proliferating cells require an increased uptake of nutrients. As hSCs can reprogram their metabolism to meet their needs, we evaluated the production/ consumption of key metabolic metabolites. One of the major roles of hSCs is to metabolize glucose to produce pyruvate and lactate, so these substrates can be used for energy production by developing germ cells. Hence, hSCs metabolism highly relies on glycolysis. Our results showed 
Table 2 Oxidative damage levels, evaluated by carbonyl groups, nitration and lipid peroxidation, of human Sertoli cells from the control group and groups exposed to 5 or $50 \mu \mathrm{M}$ of L-theanine

\begin{tabular}{llll}
\hline Oxidative damage levels & Control & $5 \mu \mathrm{M}$ of L-theanine & $50 \mu \mathrm{M}$ of L-theanine \\
\hline Carbonyl groups & $1.00 \pm 0.11$ & $1.01 \pm 0.12$ & $1.05 \pm 0.09$ \\
Nitration & $1.00 \pm 0.13$ & $1.02 \pm 0.14$ & $1.05 \pm 0.18$ \\
Lipid peroxidation & $1.00 \pm 0.11$ & $0.92 \pm 0.06$ & $0.98 \pm 0.14$ \\
\hline
\end{tabular}

Results are expressed as mean \pm SEM (fold variation to the control), $n=6$ for each condition

that the addition of a pharmacological dose of L-theanine $(50 \mu \mathrm{M})$ to hSCs culture medium triggers a higher consumption of glucose (Fig. 2A), which is the main substrate available in the culture media $(18 \mathrm{mM})$. A higher glucose uptake suggests that more pyruvate is being produced through glycolysis. We could not detect pyruvate in the extracellular media, as analyzed by the ${ }^{1} \mathrm{H}-\mathrm{NMR}$, which indicates that it is being used by the cells. Pyruvate is an important regulatory point of cell metabolism as it can follow three different pathways: (1) it can be converted to lactate by LDH; (2) it can produce alanine; or (3) it can enter the mitochondria to form acetyl-CoA and fuel the Krebs cycle [1]. Both LDH protein expression levels and activity were similar between the experimental groups, resulting in a normal production of lactate in hSCs exposed to L-theanine. Moreover, the protein expression of MCT4, a specific monocarboxylate transporter that exports lactate and pyruvate to the extracellular medium, was also normal, showing that lactate and pyruvate production and export were not altered in the presence of L-theanine. Likewise, alanine production was similar between the experimental groups (data not shown). This led us to speculate that the higher pyruvate production resulting from the higher glucose uptake was used by the mitochondria to produce metabolic intermediates necessary for anabolic processes that support the higher cellular proliferation. The fact that we did not detect acetate or citrate in the extracellular media (by the ${ }^{1} \mathrm{H}-\mathrm{NMR}$ analysis) supports that these metabolites are used to fuel the Krebs cycle, as they are exported to the extracellular compartment when they are not required for cellular processes. In fact, an increased mitochondrial membrane potential was observed in the group of hSCs exposed to the pharmacological dose of L-theanine $(50 \mu \mathrm{M})($ Fig. 3A), suggesting that L-theanine influences hSC mitochondrial function. NADH reduced coenzyme produced from glycolysis contains electrons that have a high transfer potential. These electrons are removed from NADH and passed to oxygen by the electron transport chain (ETC-mitochondrial complexes I-IV) in the mitochondrial inner membrane. So, the increased glycolytic rates of hSCs exposed to the pharmacological dose of L-theanine $(50 \mu \mathrm{M})$ induced an increased mitochondrial membrane potential. However, the protein expression of mitochondrial complexes (OXPHOS) was not altered (Table 1), neither was oxygen consumption (Fig. 3B). Still, in response to the detected metabolic changes, mitochondrial alterations could lead to an imbalanced ROS production and affect cellular proteins and lipids. However, our results show that hSCs oxidative profile was not altered by exposure to L-theanine (Table 2). This may be due to a normal mitochondrial function or the antioxidant properties attributed to this amino acid. In fact, previous studies demonstrated that L-theanine is able to increase cellular antioxidant capacity due to its structural similarity to glutamic acid, as it is also a precursor of the main endogenous antioxidant glutathione [17].

Besides glycolysis, glutaminolysis is also a very important process to maintain the high metabolic rates of hSCs. Glutamine can be incorporated by hSCs through glutamate receptors, being hydrolyzed to glutamate by the enzyme glutaminase (EC 3.5.1.2). Consequently, glutamate may be excreted, or it can be further metabolized to yield $\alpha$-ketoglutarate, a reaction-specific substrate for the Krebs cycle. L-Theanine structure is very similar to that of glutamine, thus being able to bind to glutamate receptors in cells, although with lower affinity (80-fold difference). It has been reported that in brain cells, L-theanine may exert an agonist or antagonist action on glutamate receptors in a dose- and receptor-dependent manner [18]. Glutamine is a core metabolite for tumor cell proliferation. So, the inhibition of glutamate receptors by L-theanine is under investigation in many cancer studies, to find new ways to prevent tumor cells proliferation. In the present study, if an inhibition of glutamine uptake by hSCs was occurring, we would be able to measure these metabolites in the extracellular media by the ${ }^{1} \mathrm{H}-\mathrm{NMR}$. As this was not the case and it was initially present in the extracellular media $(3 \mathrm{mM})$, it was consumed by the hSCs. Moreover, as L-theanine is a precursor of glutamate, at the pharmacological dose it seems to be an extra source of glutamate for hSCs, thus explaining why this group of cells proliferate more. Previous in vitro studies with other cells suggested that even if L-theanine cannot be metabolized through glutaminolysis, it is similarly incorporated into cell cytoplasm and can exert a glutamate-like effect [16]. A stimulation of glycolysis by glutamate was previously reported [19], as demonstrated in our study. 


\section{Conclusions}

Our data indicate that exposure of hSCs to a pharmacological dose of L-theanine $(50 \mu \mathrm{M})$ prompts an increase in cell proliferation and a higher glucose metabolization. This leads to an increased glycolytic rate to maintain the pools of Krebs cycle intermediates for ATP production and cellular components synthesis, to support the anabolic processes needed for building new cells. Although the mechanisms by which these metabolic alterations induce cell growth and proliferation remain poorly understood, the proliferative rates are correlated with substrate availability and cellular metabolism. Besides, a higher proliferation of hSCs may be important to maintain spermatogenesis and improve male reproductive potential. The glutamine-like effects of L-theanine reinforce the complementary action between glucose and glutaminolysis in hSC metabolic function. Overall, our results support that a pharmaceutical L-theanine supplementation may be used to prevent or counteract spermatogenesis disruptions caused by some health conditions.

Acknowledgements This work was supported by "Fundação para a Ciência e a Tecnologia"-FCT to Tânia R. Dias (SFRH/ BD/109284/2015); Raquel L. Bernardino (SFRH/BD/103105/2014); Marco G. Alves (IFCT2015); Pedro F. Oliveira (IFCT2015); CICS (UID/Multi/00709/2013), UMIB (PEst-OE/SAU/UI0215/2014) and REQUIMTE (UID/QUI/50006/2013). The work was co-funded by FEDER through the COMPETE/QREN, FSE/POPH (PTDC/BIMMET/4712/2014 and PTDC/BBB-BQB/1368/2014), and POCICOMPETE 2020 (POCI-01-0145-FEDER-007491) funds. The funding sources had no involvement in the study design; collection, analysis and interpretation of data; writing of the report; or in the decision to submit the article for publication.

Author contributions TRD performed most of the experiments, analyzed the results and wrote the first draft of the manuscript. RLB helped in the cell cultures and data interpretation. MGA was responsible for the metabolic analysis and helped in data interpretation and discussion. JS and $\mathrm{AB}$ collected the testicular biopsies from the patients and obtained a written consent from each of them. MS, SC, BMS and PFO designed the study, helped with statistical analysis and revised the manuscript. The manuscript was written through the contributions of all authors. All authors have given approval to the final version of the manuscript.

\section{Compliance with ethical standards}

Conflict of interest The authors declare that they have no conflict of interest.

\section{References}

1. Dias TR, Martins AD, Reis VP, Socorro S, Silva BM, Alves MG, Oliveira PF (2013) Glucose transport and metabolism in sertoli cell: relevance for male fertility. Curr Chem Biol 7(3):282-293
2. Dias TR, Alves MG, Bernardino RL, Martins AD, Moreira AC, Silva J, Barros A, Sousa M, Silva BM, Oliveira PF (2015) Dosedependent effects of caffeine in human sertoli cells metabolism and oxidative profile: relevance for male fertility. Toxicology 328:12-20

3. Dias TR, Alves MG, Silva J, Barros A, Sousa M, Casal S, Silva BM, Oliveira PF (2017) Implications of epigallocatechin-3-gallate in cultured human sertoli cells glycolytic and oxidative profile. Toxicol In Vitro 41:214-222

4. Dias TR, Alves MG, Casal S, Silva BM, Oliveira PF (2016) The single and synergistic effects of the major tea components caffeine, epigallocatechin-3-gallate and L-theanine on rat sperm viability. Food Funct 7(3):1301-1305

5. Dias TR, Alves MG, Rato L, Casal S, Silva BM, Oliveira PF (2016) White tea intake prevents prediabetes-induced metabolic dysfunctions in testis and epididymis preserving sperm quality. J Nutr Biochem 37:83-93

6. Li G, Ye Y, Kang J, Yao X, Zhang Y, Jiang W, Gao M, Dai Y, Xin Y, Wang Q (2012) L-Theanine prevents alcoholic liver injury through enhancing the antioxidant capability of hepatocytes. Food Chem Toxicol 50(2):363-372

7. Kim TI, Lee YK, Park SG, Choi IS, Ban JO, Park HK, Nam S-Y, Yun YW, Han SB, Oh KW (2009) L-Theanine, an amino acid in green tea, attenuates $\beta$-amyloid-induced cognitive dysfunction and neurotoxicity: reduction in oxidative damage and inactivation of ERK/p38 kinase and NF- $\kappa \mathrm{B}$ pathways. Free Radic Biol Med 47(11):1601-1610

8. Cho H-S, Kim S, Lee S-Y, Park JA, Kim S-J, Chun HS (2008) Protective effect of the green tea component, L-theanine on environmental toxins-induced neuronal cell death. Neurotoxicology 29(4):656-662

9. Steger K, Rey R, Kliesch S, Louis F, Schleicher G, Bergmann M (1996) Immunohistochemical detection of immature sertoli cell markers in testicular tissue of infertile adult men: a preliminary study. Int J Androl 19(2):122-128

10. Scheid L, Ellinger S, Alteheld B, Herholz H, Ellinger J, Henn T, Helfrich H-P, Stehle P (2012) Kinetics of L-theanine uptake and metabolism in healthy participants are comparable after ingestion of L-theanine via capsules and green tea-4. J Nutr 142(12):2091-2096

11. Oliveira PF, Alves MG (2015) The sertoli cell at a glance. Sertoli cell metabolism and spermatogenesis. Springer, New York, pp 3-13

12. Oliveira P, Alves M, Rato L, Silva J, Sa R, Barros A, Sousa M, Carvalho R, Cavaco J, Socorro S (2011) Influence of $5 \alpha$-dihydrotestosterone and $17 \beta$-estradiol on human sertoli cells metabolism. Int J Androl 34(6pt2):e612-e620

13. Oliveira P, Alves M, Rato L, Laurentino S, Silva J, Sa R, Barros A, Sousa M, Carvalho R, Cavaco J (2012) Effect of insulin deprivation on metabolism and metabolism-associated gene transcript levels of in vitro cultured human sertoli cells. Biochim Biophys Acta Gen Subj 1820 2:84-89

14. Alves MG, Socorro S, Silva J, Barros A, Sousa M, Cavaco JE, Oliveira PF (2012) In vitro cultured human sertoli cells secrete high amounts of acetate that is stimulated by $17 \beta$-estradiol and suppressed by insulin deprivation. Biochim Biophys Acta Mol Cell Res 1823 8:1389-1394

15. Reis MM, Moreira AC, Sousa M, Mathur PP, Oliveira PF, Alves MG (2015) Sertoli cell as a model in male reproductive toxicology: advantages and disadvantages. J Appl Toxicol 35(8):870-883

16. Takarada T, Nakamichi N, Nakazato R, Kakuda T, Kokubo H, Ikeno S, Nakamura S, Kuramoto N, Hinoi E, Yoneda Y (2016) Possible activation by the green tea amino acid theanine of mammalian target of rapamycin signaling in undifferentiated neural progenitor cells in vitro. Biochem Biophys Rep 5:89-95 
17. Vuong QV, Bowyer MC, Roach PD (2011) L-Theanine: properties, synthesis and isolation from tea. J Sci Food Agric 91(11):1931-1939

18. Kakuda T, Yanase H, Utsunomiya K, Nozawa A, Unno T, Kataoka K (2000) Protective effect of $\gamma$-glutamylethylamide (theanine) on ischemic delayed neuronal death in gerbils. Neurosci Lett 289(3):189-192

19. Loaiza A, Porras OH, Barros LF (2003) Glutamate triggers rapid glucose transport stimulation in astrocytes as evidenced by realtime confocal microscopy. J Neurosci 23(19):7337-7342 